\title{
In vivo assessment of local effects after application of bone screws delivering bisphosphonates into a compromised cancellous bone site
}

\author{
Alireza Roshan-Ghias a, Joerg Arnoldi ${ }^{\text {b,c }}$, Philip Procter ${ }^{\text {b,d }}$, Dominique P. Pioletti a,* \\ ${ }^{a}$ Laboratory of Biomechanical Orthopedics, Institute of Bioengineering, Ecole Polytechnique Federale de Lausanne (EPFL), Switzerland \\ b Stryker Osteosynthesis, Switzerland \\ ' Institute for Clinical Science, Sahlgrenska Academy, University of Gothenburg, Sweden \\ d School of Engineering and Design, Brunel University, Uxbridge, UK
}

\section{A R T I C L E I N F O}

\section{Article history:}

Received 5 April 2011

Accepted 1 June 2011

\section{Keywords:}

Bone screws

Cancellous bone

Drug delivery

Biomechanics

Histology

\begin{abstract}
A B S T R A C T
Background: The primary stability of cancellous screw is difficult to obtain in bone of compromised quality and failure of screw fixation is common. To overcome this problem, it is proposed to locally deliver bisphosphonate from the screw. An in vivo validation of the increase in fixation of the cancellous screw is then needed in compromised bone.

Methods: In this study, we used an overdrilling procedure, which enables consistent modeling of reduced screw stability comparable to compromised cancellous bone. Forty eight adult NZW rabbits were used in this study and all animals underwent bilateral femur implantation. One leg was implanted with the screw containing the bisphosphonate (biocoated group) while the other was used as control (control group) with the screw only. Mechanical testing and micro-CT imaging were used to assess the effect of local drug delivery of Zoledronate on screws fixation at 5 time points.

Findings: At the early time points (1,5, and 10 days), no significant difference could be seen between the biocoated and control groups. At 6 weeks, the bone volume fraction was significantly higher in the trabecular region of the biocoated group. However, this increase did not have a significant effect on the pull-out force. At the last time point, 11 weeks, both the bone volume fraction and the pull-out force were significantly higher in the biocoated group.

Interpretation: The results of this study suggest that, in compromised bone, local delivery of bisphosphonate enhances the stability of bone screws.
\end{abstract}

(C) 2011 Elsevier Ltd. All rights reserved.

\section{Introduction}

Initial fixation of screws in osteoporotic bone is frequently difficult to obtain (Seebeck et al., 2005). Moreover, during the early healing phase, micromotions between implant and bone can activate an osteoclastic response, which may increase further bone resorption (Stadelmann et al., 2008) and consequently may lead to unstable fixation. Indeed, there is a clinically observed high rate of failure of screw fixation in osteoporotic patients (Bjorkenheim et al., 2004).

Bisphosphonates, predominantly amino-bisphosphonates such as zoledronate are known to be highly potent drugs affecting bone metabolism by affecting osteoclast activity through the inhibition of the enzyme farnesyl pyrophosphate synthase (Russell et al., 2008). Thus one might hypothesize that the association between an orthopedic implant and a bisphosphonate could decrease the periimplant bone resorption, and hence increasing the stability of the implant. Indeed, orthopedic implants designed to locally deliver

\footnotetext{
* Corresponding author at: Laboratory of Biomechanical Orthopedics, Station 15, EPFL, 1015 Lausanne, Switzerland.

E-mail address: dominique.pioletti@epfl.ch (D.P. Pioletti).
}

bisphosphonates have been demonstrated to increase their fixation in animal studies (Wermelin et al., 2008a), even in osteoporotic bone (Peter et al., 2006) or very recently for dental implant in human (Abtahi et al., 2010). Several reviews have been recently published supporting the idea that a general positive effect is observed on bone volume fraction and implant fixation when an implant is used as local bisphosphonate delivery (Aspenberg, 2009; Pioletti et al., 2008). In particular, increase in bone volume fraction and pull-out force was observed as early as 2 to 3 weeks in rats after insertion of the implant coated with bisphosphonate (Peter et al., 2005; Wermelin et al., $2008 \mathrm{~b}$ ). The increase in implant fixation was correlated to the amount of bone formed at the close vicinity of the implant (Peter et al., 2006). However, as observed by Aspenberg, the local presence of the bisphosphonate is not responsible for new bone formation, but rather for the preservation of the initial osteoid formed around the implant which can then be used as scaffold for further bone formation (Aspenberg, 2009) and finally leading to an increase implant fixation.

The coating of screw with bisphosphonate in osteoporotic bone would then be an advantageous solution and could help in the screw fixation. However, available animal models intended to model human osteoporosis suffer from certain drawbacks (Turner, 2001). Even after 
combining different strategies such as ovariectomy, low-calcium diet and steroid application, in sheep for example, bone densities have been demonstrated to be significantly higher $(\geq 30 \%)$ than healthy human bone equivalents (Augat et al., 2003). Currently, osteoporotic animal models failed to reach bone density levels being obvious in human osteoporotic bone. In parallel, osteoporotic bone is also characterized by a reduction of trabeculi number as well as reduced thickness (Parfitt et al., 1983). The impaired trabecular architecture results in smaller contact area between implant and bone, which in the case of orthopedic screws, is similar to an increase in gap between the core of the screw and the bone. It has then been proposed to use an overdrilling defect to create oversized defect with a low implant/bone contact area. The intention of this model was to create a reduced pullout force being comparable to data achieved in osteopenic or osteoporotic bone (Hoshikawa et al., 2003).

In this study we hypothesize that local delivery of bisphosphonate on screw results in enhanced fixation in compromised cancellous bone. For this purpose, an overdrilling procedure was used, which enables consistent modelling of reduced screw stability comparable to compromised cancellous bone.

\section{Methods}

This study was conducted in accordance with the provisions of the FDA Good Laboratory Practice (21 CFR, Part 58, April 01, 2006) and the OECD Good Laboratory Practices, (ENV/CM/CHEM (98)11, 1997). The study plan was approved by the Biomatech Ethical Committee on November 19, 2009.

\subsection{Animals}

Forty eight adult 4 month old NZW rabbits were randomly divided into 4 groups of $n=10$ each and one reserve group of $n=8$. Husbandry conditions were in conformance to the European requirements (Directive EEC/86/609). The rabbits were individually housed. Humidity was maintained higher than $30 \%$ of relative humidity and temperature was between $17{ }^{\circ} \mathrm{C}$ and $21^{\circ} \mathrm{C}$. The artificial light cycle was controlled using an automatic timer ( $12 \mathrm{~h}$ of light, $12 \mathrm{~h}$ of dark). Municipal water was delivered ad libitum. Rabbit granulated high fiber diet 115 (SAFE, Augy, France) was provided daily, $200 \mathrm{~g}$ per day. All rabbits underwent bilateral femur implantation. In a paired approach, each medial distal femoral condyle received one $4.0 \mathrm{~mm} \emptyset$ cancellous bone screw, either coated with zoledronate-loaded fibrinogen (hereafter termed biocoated screws) or non-coated (control screws). The implantation site as well as the application of biocoated and control screws was randomized. As no animal replacement was needed during the observation period, the reserve animals were used to create another long-term follow-up (11 weeks). Samples were analyzed by means of micro-CT, biomechanical testing and histology.

\subsection{Implants}

For implantation, a standard $4.0 \mathrm{~mm} \varnothing$ Titanium bone screw (bone screw \#604014, Stryker, Selzach, Switzerland), intended for use in cancellous bone was used. The core diameter of the screw was $1.9 \mathrm{~mm}$ and the corresponding standard drill diameter $2.5 \mathrm{~mm}$. The overall screw length of $14 \mathrm{~mm}$ was defined as the intended insertion depth of $10 \mathrm{~mm}$ plus a sufficient distance of the screw head above the bone surface to enable clamping the screw head for the biomechanical testing. Sixty bone screws were provided to AddBIO AB (Linköping, Sweden) for biocoating (biocoated group) (proprietary name FibMat). Another 60 bone screws served as controls (control group).

\subsection{Biocoating with bisphosphonate}

Bisphosphonate (synthetic zoledronic acid, REF ALX-430-153M025) was provided by the chemical supplier Alexis (Lausen, Switzerland). Screws were coated with a fibrinogen multilayer incorporating an amount of $150 \mathrm{ng} / \mathrm{cm}^{2}$ of zoledronic acid (Tengvall et al., 2004). This loading dose could be considered comparable to a local concentration of $500 \mathrm{nM}$. As a comparison, the half maximum inhibitory concentration $\left(\mathrm{IC}_{50}\right)$ for zoledronate is about $5 \mathrm{nM}$. After single packaging in peel-pouches, all screws were sterilized by performing $\gamma$-irradiation at 17-25 kGy (Leoni, Switzerland).

\subsection{Surgical procedure}

In a standardized procedure, the femoral condyle was addressed for implantation. Surgical as well as implantation procedure followed a previously published protocol (Hoshikawa et al., 2003). The surgical approach was done bilaterally by performing one implantation per site. The "paired" design allowed comparison between biocoated and control groups and reduced variation. A longitudinal incision was performed demonstrating the medial aspect of each distal femoral condyle. The entry point for drilling was localized by using the insertion point of the medial contralateral ligament as a landmark. Going $5 \mathrm{~mm}$ proximal, the entry point for drilling was set. Direction of drilling was maintained perpendicular to the slightly curved bone surface. Any perforation of the intracondylar groove was avoided.

Drilling was initiated by introducing a $1.4 \mathrm{~mm} \varnothing$ Guide Wire. To avoid perforation of the opposite cortex, the Guide Wire was labeled with a laser marker at a $10 \mathrm{~mm}$ insertion depth. Allowing appropriate allocation of the defect, opening to final drill size was performed using a cannulated $3.7 \mathrm{~mm} \varnothing$ drill. The choice of a $3.7 \mathrm{~mm}$ overdrill diameter was based on a preliminary study made in cadaver knee rabbit showing that this diameter effectively decreased the pull-out force (data not shown). The customized drills were equipped with a depth stop at $10 \mathrm{~mm}$. To reduce thermal impact of drilling and remove bone debris, during and after power-driven drilling, flushing with saline solution was performed. The screw was first inserted by hand, then turned down to final position using the screwdriver. The hind limb wound was closed by subcutaneous and cutaneous suturings. A systemic antibiotic and pain management was applied. Anteroposterior (AP) and mediolateral (ML) radiographs were taken to document screw positioning. The rabbits were monitored until complete recovery from the anesthesia and then returned to their cages.

\subsection{Sample preparation/data analysis}

All animals were kept under observation for the proposed time points. The animals were observed daily for general health and in particular for any visible sign of screw migration, wound infection or major signs of pain. At termination, the animals were euthanized under anesthesia (intramuscular injection of Zoletil ${ }^{\circledR}$, Virbac, France) by intravenous pentobarbital injection (Dolethal ${ }^{\circledR}$, Vetoquinol, France). At 1, 5 and 10 days as well as at 6 and 11 weeks, animals were sacrificed and the femoral condyles were harvested for micro-CT examination and biomechanical testing. In addition at 6 weeks, histological analysis was performed.

\subsection{Macroscopical evaluation}

At euthanasia, the region of the initial implantation was carefully exposed. Gross photography of the implant site was taken (Fig. 1). Prior to all destructive investigations, radiological analysis (AP and MD) was used to evaluate the performance up to 11 weeks. 

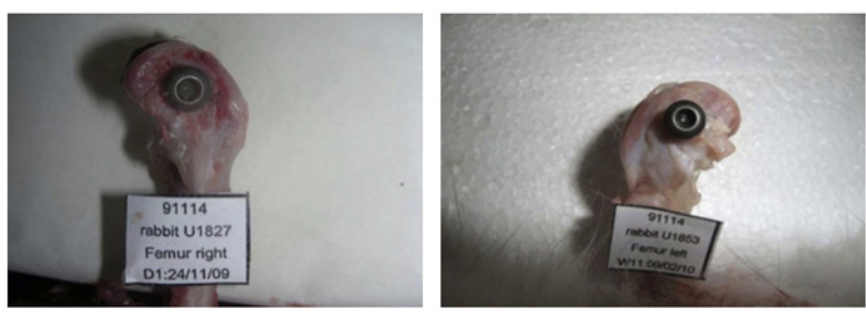

Fig. 1. Implantation site after (left) 1 day (control) and (right) 11 weeks (biocoated)

\subsection{Micro-CT imaging}

The screw head was clamped in a specially designed screw holding sleeve to ensure the alignment of the screw with the scanning axis. The holding sleeve was then placed in the polystyrene support of the in-vivo MCT Scanner 1076 (SkyScan, Belgium). Samples were scanned at $100 \mathrm{kV} / 100 \mathrm{~mA}$ source voltage/current, with a $1 \mathrm{~mm}$ aluminum filter. The pixel size (resolution), rotation step, and exposure time were $18 \mu \mathrm{m}$, $0.6^{\circ}$ over $360^{\circ}$, and $400 \mathrm{~ms}$, respectively. The dataset was reconstructed with NRecon software (SkyScan, Belgium). Moderate beam hardening was applied in the reconstruction process. The $\mu \mathrm{CT}$ analysis was done with dedicated software CTAN (SkyScan, Belgium). The Hounsfield Unit (HU) and bone mineral density (BMD) calibrations were first applied to the dataset. Two phantoms of calcium density $0.25 \mathrm{~g} / \mathrm{mm}^{3}$ and $0.75 \mathrm{~g} /$ $\mathrm{mm}^{3}$ of $8 \mathrm{~mm}$ diameter (Gloor Instruments, Switzerland) were scanned in a $10 \mathrm{ml}$ Falcon tube filled with purified water using the scanning parameters described previously. Manufacturer's HU and BMD calibration procedures were then followed. The region of interest (ROI) was chosen as a sleeve around the screw with $0.5 \mathrm{~mm}$ of thickness (Fig. 2). The cortical bone at the entrance point of screw was excluded. Afterwards, the bone volume (BV), tissue volume (TV), and BMD of bone tissue were measured for each dataset. Bone volume fraction (BVF) is defined as the percentage of bone volume over tissue volume (\%BV/ TV).

\subsection{Biomechanical analysis}

Implant sites were prepared for biomechanical analysis by wrapping them in isotonic drained (PBS) patches, packed into labeled tubes and, stored in a cooled environment $\left(4^{\circ} \mathrm{C}\right)$. For each time point, 12 samples $(6 \times$ biocoated and $6 \times$ control) were evaluated. The specimens were embedded in Neukadur cement (Troller, Switzerland) keeping the screw axis perpendicular to the mold, making sure the later pull-out orientation was always consistent. The screw head was then gripped and subjected to pull-out (Instron, USA) until bone failure. The maximum pull-out force was measured for each sample, accordingly.
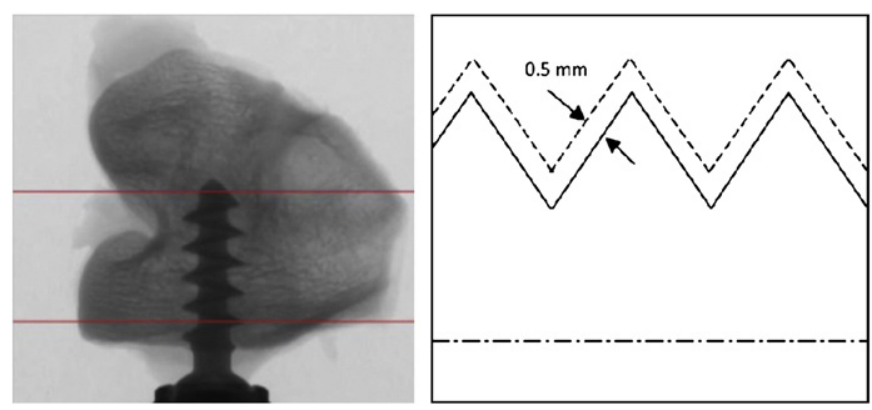

Fig. 2. (Left) X-Ray photo of screw embedded in distal femur. Red lines indicate the area of interest. (Right) Illustration showing the ROI around the threads. The dash dot line represents the axis of the screw.

\subsection{Histology}

Samples were collected at 6 weeks after implantation $(4 \times$ biocoated and $4 \times$ control, one section was prepared out of each sample and evaluated). The implant sites were removed as small blocks by sawing perpendicular to the screw canals. Subsequently, the blocks were fixed in $4 \%$ paraformaldehyde for up to 1 week, dehydrated in a series of increasing concentrations of ethanol and embedded in polymethylmethacrylate according to standard histological techniques. Two longitudinal sections per specimen were obtained by micro-cutting and grinding procedures (Donath and Breuner, 1982). The ground sections (20 to $30 \mu \mathrm{m}$ ) were stained with modified paragon for qualitative and semi-quantitative analyses.

\subsection{Statistical analysis}

All statistical analysis was performed in MATLAB (Mathworks). Group comparisons were done using two-way ANOVA. As the control screws were paired with the biocoated screws in each rabbit, we employed paired t-test for pair-wise comparison. Biomechanical differences were considered significant if $P<0.05$.

\section{Results}

\subsection{Macroscopic evaluation}

Transverse slices of samples from all time points are shown in Fig. 3. As the holes were over-drilled prior to the insertion of the screws, it was expected that there would be little contact between screw and bone at earlier time points ( 1 day, 5 days, and 10 days). Nevertheless, in a few samples some bone was observed in the internal thread (Fig. 3a). This might be the residue from the drilling process. Also notice that in some cases, the screw is not concentric with the hole (Fig. 3b). These two factors may play an important role in the variation between samples within the same time point. At 6 weeks, bone formation around the walls of the internal thread (Fig. $3 \mathrm{~d}$ and i) is clearly observed, and at 11 weeks, bone has covered the internal thread completely (Fig. 3e and $\mathrm{j}$ ). Also note the formation of callus around the external part of some of the screws at 11 weeks (Fig. 3e).

\subsection{Micro-CT imaging}

BVF was measured in a defined ROI around the screw. During the pull-out tests it was observed that the amount of bone tissue taken out with the screw increases over time. This observation was confirmed by

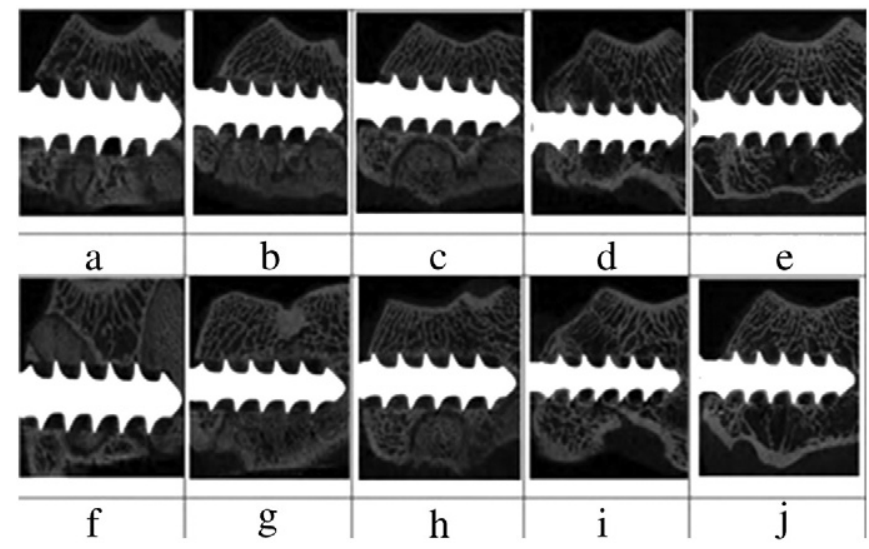

Fig. 3. Transverse slices of distal femur through the screw for a typical rabbit at each time point. (a)-(e) Control group, and (f)-(j) biocoated group, at 1 day, 5 days, 10 days, 6 weeks, and 11 weeks, respectively. 
measuring BVF over time, which significantly increases $(P<0.01)$ (Fig. 4a). The BD in the biocoated group is significantly higher at 6 and 11 weeks compared to the control group $(P<0.01$ and $P=0.015$, respectively), but not at the early time points. It was noted that BMD of bone tissue in ROI significantly increased in time in each group, but there was no difference between the two groups.

\subsection{Biomechanical analysis}

The biocoated group had a significantly higher pull-out force at 11 weeks than the control group $(P=0.05)$ but not at prior time points (Fig. 4b). The correlation between $\mathrm{BD}$ and maximum pull-out force was 0.95 .

\subsection{Histology}

Analyzing control and biocoated groups six weeks after implantation, a consistent osseous reaction was noticed. Bone ingrowth showing signs of remodeling was observed within the screw threads of both groups. No residue of fibrin, blood clots, fibroblastic tissue or bone debris but a differentiated adipocytic bone marrow could be detected further substantiating the ongoing remodeling process as shown for a biocoated sample in Fig. $5 a$ and $b$. The cartilaginous tissue associated with ossification areas was fully ossified with formation of a young cancellous bone (Fig. $5 \mathrm{c}$ and d). At 6 weeks, histological sections of the biocoated group demonstrated extensive bone formation covering the screw thread almost completely.

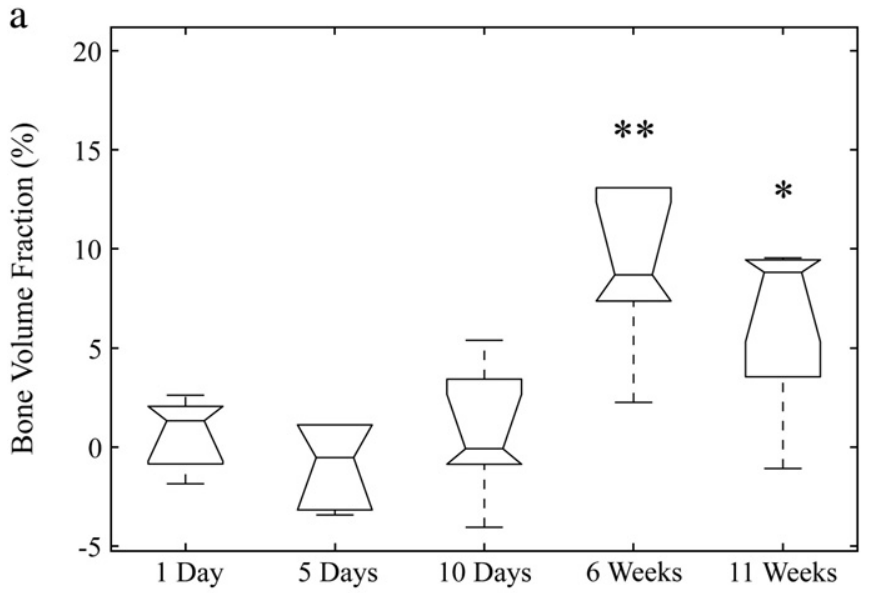

$\mathrm{b}$

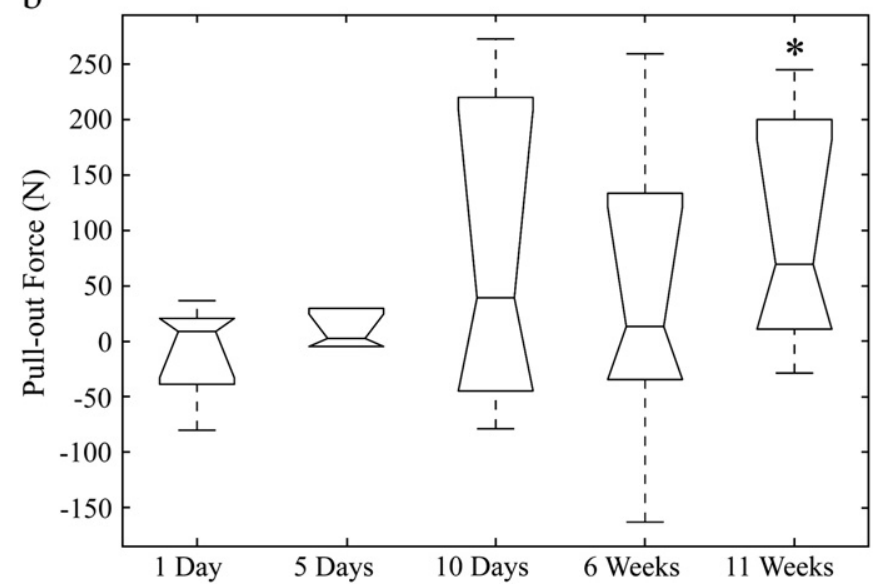

Fig. 4. Box plot of the paired difference between coated and control group (left) bone volume fraction, and (right) pull-out force, at different time points ( ${ }^{*}: P$-value $<0.05$, **: $P$-value $<0.01$ )
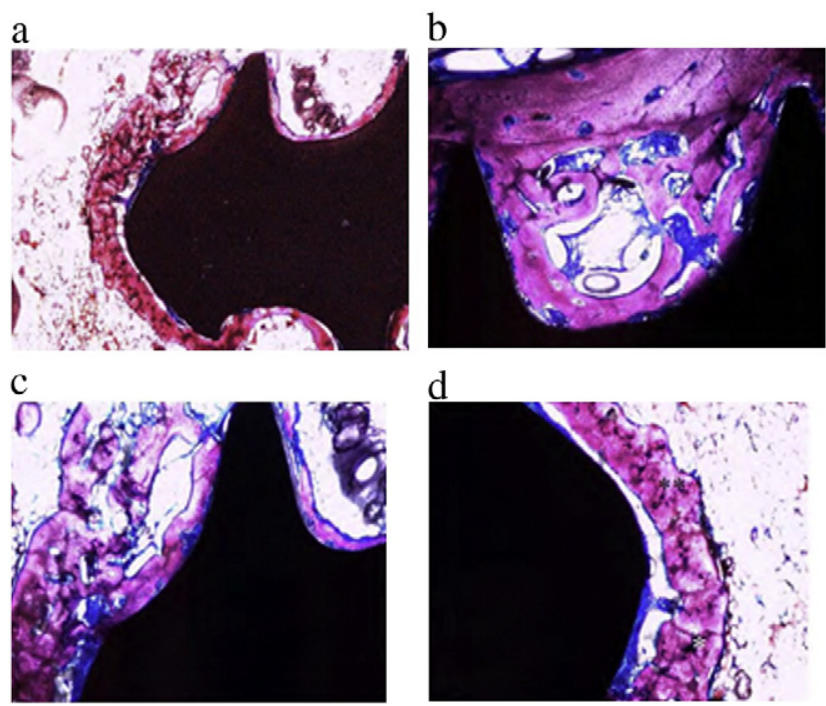

d

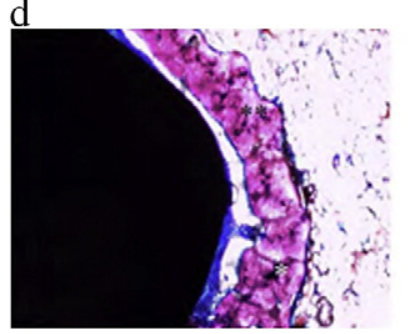

Fig. 5. At 6 weeks for a biocoated sample, bone formation around the walls of the internal thread started to cover the internal thread almost completely. (a) Screw tip is completely "coated" by new bone and (b) screw geometry almost completely covered by newly formed bone. (c) Formation of trabecular like bone in the empty space around the screw. (d) The cartilaginous tissue (marked by *) was fully ossified with formation of young bone (marked by ${ }^{* *}$ ) (for scale, the distance between two thread is $1.75 \mathrm{~mm}$ ).

\section{Discussion}

The objective of this study was to evaluate the effect of local bisphosphonate application on screw fixation in compromised cancellous bone. To reduce screw stability comparable to compromised cancellous bone, an overdrilling procedure was conducted. Three early time points ( 1 day, 5 days, and 10 days) and two late time points ( 6 and 11 weeks) were chosen for the evaluation. This study showed that at the last time point, both the bone volume fraction and the pull-out force were significantly higher in the biocoated group. Thus our hypothesis was verified. The local delivery of bisphosphonate on screw results in enhanced fixation in compromised cancellous bone.

Developing new strategies for achieving stable fracture fixation in low-density cancellous bone requires establishing appropriate preclinical in vivo models. A limited number of options were available. Sheep are one of the dominant orthopedic animal models and have been frequently used to investigate the impact of compromised bone on implant stability and performance. Osteopenic sheep or even goat demonstrated a significant reduction in bone mineral density and trabecular micro architecture as compared to healthy ones (Schorlemmer et al., 2003). Reduction of bone mineral density reaches levels of $200-300 \mathrm{mg} / \mathrm{cm}^{3}$. Although bone mineral density is significantly reduced, levels, seen in human osteoporotic patients $\left(50-150 \mathrm{mg} / \mathrm{cm}^{3}\right.$ ) are not reached (Riggs et al., 2004). Another option, intended to simulate insufficient bone mass is the technique of overdrilling used in the present study with rabbit. Originally this technique was developed to demonstrate the beneficial effects of bone augmentation with bone cements. Hoshikawa et al. showed that overdrilling screw sites allowed demonstration of the effects of screw augmentation even in bone being more dense than comparable human bone structure sites (Hoshikawa et al., 2003). In recent literature, mechanical testing in bone with a low bone mineral density either induced by osteoporosis or by overdrilling is claimed to result in a similar effect (Grardel et al., 1994; Castaneda et al., 2008). The model mimics a low bone density by providing less bone/implant contact. The overdrilling model has certain limitations. However, the overdrilling model does change the trabecular density as well as the 
trabecular thickness, both being characteristic critical parameters in osteoporotic human bone (Parfitt et al., 1983).

Based on the current literature (Peter et al., 2005; Wermelin et al. 2008a), we hypothesized that local delivery of bisphosphonate enhances the fixation of the screw in a compromised cancellous site. This hypothesis was verified although only at the latest time point. The present study provided no evidence that local drug application at the implantation site had a significant impact on osteoclastic activity. The histological evaluation of biocoated and control implant sites revealed no significant differences in the resorption pattern. This might be related to the low dose application of zoledronate or also to the increase in diffusion path of the drug generated by the overdrilling procedure. The increase in diffusion path of the drug could explain that the increase in fixation was only observed at late time point.

It is noteworthy to mention the possible sources of variation in this experiment. In the CT slices, it was observed that screw orientation was not always consistent. This might be due to the overdrilling procedure, allowing a certain freedom of screw positioning during insertion. In addition, at late time points, sporadic bone formation at the exterior of the holes was obvious in both groups, impacting the outcome of the pull-out testing.

\section{Conclusion}

The results of this study suggest that local delivery of bisphosphonate enhances the stability of bone screws in the rabbit model. However, further dose response evaluations will be needed for analyzing the effects of different local drug concentrations released from biocoatings on metal implants.

\section{Acknowledgment}

The FibMat (fibrinogen) coated screws were kindly provided by $\mathrm{Dr}$ Trine Vikinge, AddBIO AB, Linköping Sweden. This study was partially funded by Stryker Osteosynthesis, Selzach, Switzerland.

\section{References}

Abtahi, J., Tengvall, P., Aspenberg, P., 2010. Bisphosphonate coating might improve fixation of dental implants in the maxilla: a pilot study. Int. J. Oral Maxillofac. Surg. 39, 673-677.

Aspenberg, P., 2009. Bisphosphonates and implants: an overview. Acta Orthop. 80, 119-123.

Augat, P., Schorlemmer, S., Gohl, C., Iwabu, S., Ignatius, A., Claes, L., 2003. Glucocorticoid-treated sheep as a model for osteopenic trabecular bone in biomaterials research. J. Biomed. Mater. Res. A 66, 457-462.
Bjorkenheim, J.M., Pajarinen, J., Savolainen, V., 2004. Internal fixation of proximal humeral fractures with a locking compression plate: a retrospective evaluation of 72 patients followed for a minimum of 1 year. Acta Orthop. Scand. 75, 741-745.

Castaneda, S., Calvo, E., Largo, R., Gonzalez-Gonzalez, R., De La Piedra, C., Diaz-Curiel, M., et al., 2008. Characterization of a new experimental model of osteoporosis in rabbits. J. Bone Miner. Metab. 26, 53-59.

Donath, K., Breuner, G., 1982. A method for the study of undecalcified bones and teeth with attached soft tissues. The Sage-Schliff (sawing and grinding) technique. J. Oral Pathol. 11, 318-326.

Grardel, B., Sutter, B., Flautre, B., Viguier, E., Lavaste, F., Hardouin, P., 1994. Effects of glucocorticoids on skeletal growth in rabbits evaluated by dual-photon absorptiometry, microscopic connectivity and vertebral compressive strength. Osteoporos. Int. 4, 204-210.

Hoshikawa, A., Fukui, N., Fukuda, A., Sawamura, T., Hattori, M., Nakamura, K., et al., 2003. Quantitative analysis of the resorption and osteoconduction process of a calcium phosphate cement and its mechanical effect for screw fixation. Biomaterials 24, 4967-4975.

Parfitt, A.M., Mathews, C.H., Villanueva, A.R., Kleerekoper, M., Frame, B., Rao, D.S., 1983. Relationships between surface, volume, and thickness of iliac trabecular bone in aging and in osteoporosis. Implications for the microanatomic and cellular mechanisms of bone loss. J. Clin. Invest. 72, 1396-1409.

Peter, B., Pioletti, D.P., Laib, S., Bujoli, B., Pilet, P., Janvier, P., et al., 2005. Calcium phosphate drug delivery system: influence of local zoledronate release on bone implant osteointegration. Bone 36, 52-60.

Peter, B., Gauthier, O., Laib, S., Bujoli, B., Guicheux, J., Janvier, P., et al., 2006. Local delivery of bisphosphonate from coated orthopedic implants increases implants mechanical stability in osteoporotic rats. J. Biomed. Mater. Res. A 76, 133-143.

Pioletti, D.P., Gauthier, O., Stadelmann, V.A., Bujoli, B., Guicheux, J., Zambelli, P.Y., et al., 2008. Orthopedic implant used as drug delivery system: clinical situation and state of the research. Curr. Drug Deliv. 5, 59-63.

Riggs, B.L., Melton III, L.J., Robb, R.A., Camp, J.J., Atkinson, E.J., Peterson, J.M., et al., 2004. Population-based study of age and sex differences in bone volumetric density, size, geometry, and structure at different skeletal sites. J. Bone Miner. Res. 19, 1945-1954.

Russell, R.G., Watts, N.B., Ebetino, F.H., Rogers, M.J., 2008. Mechanisms of action of bisphosphonates: similarities and differences and their potential influence on clinical efficacy. Osteoporos. Int. 19, 733-759.

Schorlemmer, S., Gohl, C., Iwabu, S., Ignatius, A., Claes, L., Augat, P., 2003. Glucocorticoid treatment of ovariectomized sheep affects mineral density, structure, and mechanical properties of cancellous bone. J. Bone Miner. Res. 18, 2010-2015.

Seebeck, J., Goldhahn, J., Morlock, M.M., Schneider, E., 2005. Mechanical behavior of screws in normal and osteoporotic bone. Osteoporos. Int. 16 (Suppl 2), S107-S111.

Stadelmann, V.A., Terrier, A., Pioletti, D.P., 2008. Microstimulation at the bone-implant interface upregulates osteoclast activation pathways. Bone 42, 358-364.

Tengvall, P., Skoglund, B., Askendal, A., Aspenberg, P., 2004. Surface immobilized bisphosphonate improves stainless-steel screw fixation in rats. Biomaterials 25 , 2133-2138.

Turner, A.S., 2001. Animal models of osteoporosis-necessity and limitations. Eur. Cell Mater. 1, 66-81.

Wermelin, K., Aspenberg, P., LinderbäCk, P., Tengvall, P., 2008a. Bisphosphonate coating on titanium screws increases mechanical fixation in rat tibia after two weeks. J. Biomed. Mater. Res. A 86, 220-227.

Wermelin, K., Suska, F., Tengvall, P., Thomsen, P., Aspenberg, P., 2008b. Stainless steel screws coated with bisphosphonates gave stronger fixation and more surrounding bone. Histomorphometry in rats. Bone 42, 365-371. 